\title{
Register rerum ad Vol. 2
}

Fecit E. Flückiger, Basel R=Critical Review - Übersichtsreferate - Revues générales

Adaptation, lack of- (Homo), 223 Alimentation de vieillards en Suisse

(ville et montagne), 321 Alkoholverbrauch, täglicher, und Se-

rum-Cholesterin (Homo), 360 Aorten-Elastizität, Druck-Volumen-

Diagramm (Homo), 253 Aorten-Modell, Druck-Volumen-Dia-

gramm, Vergleich: Aorta (Homo),

253 Arginin, Gehaltsänderungen im Rippen-

knorpel (Meerschweinchen), 156 Atherosklerosis, Experimentelle (Kanin-

chen), 247 Bergbevölkerung, Nahrungsaufnahme,

Vergleich mit Städter, 321 Blood pressure, response to vasopressin,

histamine, noradrenaline (rat), 47 Blutdruck: -, Änderungen unter psychischer Bela-

stung (Homo), 223 -, basaler und Labilität (Ratte), 47 -, Veränderungen im Na- und Wasser-

haushalt (Ratte), 47

-Volumdiagramm, Aorta (Homo), 253

-wellen III. Ordnung bei psychischer Belastung (Homo), 223

Blutlipoide, Fettinfusionen, exper. Atherosklerosis, Corticoide (Kaninchen), 247

Bread, life span, bone density and body weight (rat), 185

Calcium45, turnover, tendons in thermic contraction (rat) , 11, 113

Cartilage, costal, changes in chemical composition (guinea pig), 156

Cholesterin im Serum, alte Brauerei-arbeiter, 360

Collagen:

- and hexosamine in connective tissue (Homo), 59

-, changes in -, and function of aorta (Homo), 253

-, fibres, affinity for Ca4S, 11,113; Na24, K42, I131, 113

-, Struktur der Rattenschwanzsehne, 81

-, Transformation and thermic contraction, 11

Connective tissue:

-, changes and urinary steroids (Homo) , 59

-, changes in -, and changes in function of human aorta, 253

Coronaires, lesions des - , et cholesterol sérique, 360

Creatinine, excretion (rat), 123

Diskrimination:

-, gustatorische (Homo), 104

-, visuelle (Homo), 1

DNA im entzündlichen Vorgang (Ratte), 174

Dystrophie musculaire senile et vitamine E (rat), 139

Eiweiß:

-, Ausscheidungdurch Niere (Ratte), 33

-, Gehalt im Rippenknorpel (Meerschweinchen), 156 
Electrocardiogram, changes of - (rat), 204

Electrolytes, metabolism of water and -, influence on blood pressure (rat) , 47.; urinary excretion (rat), 123

Fat, intake in rural and urban population (Switzerland), 321

Futter-Restriktion, Schwanzregenera-tion und - (Pisces), 266

\section{1/1958}

Register rerum

373

Gehörschwelle, reine Tone, exponen-tielle Abnahme (Homo) ,311

Geschmack, Schwellen für süß und salzig (Homo), 104

Glucuronic acid in costal cartilage (guinea pig), 156

Guinea pig, costal cartilage, chemical changes of -, 156

Harnsäure, Ausscheidung (Ratte), 33

Heart:

-, decrease of- rate (rat), 204

-, electrocardiogram (rat), 204

-, position changes (rat), 73

-, ventricle weight (rat), 204

Hexosamine:

-, (tissu conjonctif) et stéroïdes urinaires (Homo), 59

-, inflammations et variation de - (rat), 174

Homo:

-, alcohol intake and serum cholesterol, 360

-, Aortenelastizität, 253

-, Bindegewebe und Steroidausschei-dung, 59

-, Blutdruckänderungen unter psychi-scher Belastung, 223

-, distracting signals and performance, 136

-, Food intake, urban and mountain populations, 321

-, Gehörschwelle, reine Tone, 311

-, Nahrungsverbrauch, 290 (R), 321

-, perception and recognition, sweet and salty, 104

-, ponderal index, correlation with serum cholesterol, 360

-, Reaktionszeit, optische Reize, 284

-, Serum-Cholesterin, 360

-, seuil auditoire pour sons purs, 311

-, thresholds of hearing, 311

-, uncertainty and performance, 284

-, visuelle Reizdauer und Perzeption, 1

-, vitamin B12 deficiency and hypo-chlorhydria, 213

Hypochlorhydría as cause of vitamin B12 deficiency (Homo), 213

Hypophyse, vergrößerte, Sexualdiffe-renz (Ratte), 185

Inflammation, acute, biochemistry of (rat), 174

Iodine131, collagen fibres, affinity for -, 113

Kaninchen, Fettinfusionen und exper.

Atherosklerosis, 247 Knorpel, Rippen-, chemisette Verände- 
rungen (Meerschweinchen), 156 Konzentrationsvermögen und unterbro-

chene audit. Stórsignale (Homo), 136 Kreatin und Kreatinin im Harn und

Vitamin E (Ratte), 139 Lapin, athérome experimental, 247 Lebistes reticulatus (Pisces),

Schwanz-

regeneration, 266 Life span, on different diets (rat), 185 Lunge: -, Erkrankungen und veränderte

Herz-

lage (Ratte), 73 -, Erkrankung und Harnausscheidung

(Ratte), 123 Margarine in rat diets, lack of difference

between -, and butter, 185 Metabolism:

- $\quad$ and tail regeneration in Leb. ret.

(Pisces), 266

-, food uptake and faeces production

(rat), 21 Nahrungsverbrauch (Homo), 290 (R),

321 Nourriture:

- $\quad$ (Homo), 290 (R)

-, choisie par des personnes âgées, 321

- $\quad$ et regeneration (Pisces), 266

Perception threshold of- (Homo), 311

Performance:

- $\quad$ and distraction (auditory signals)

(Homo), 136

-, influence of uncertainty on (Homo) ,284 Periarteriitis, fehlende bei Kartoffeldiät

(Ratte), 185 Periodicity, male sexual behaviour (rat) ,

64 Phospholipids, infusion and exper. atherosclerosis (rabbit), 247 Pisces, Schwanzregeneration,

Ge-

schlechtsunterschiede bei -, Einfluß

von Retardierung bei -, 266 Polysaccharides, acid -, and aldopoly-

saccharides in costal cartilage (guinea

pig), 156 Ponderal Index, correlation with age

and serum cholesterol (Homo), 360 Potassium42, collagen fibres, affinity

for-, 113 Potatoes in diet and periarteriitis (rat),

185

374

Register rerum

11/1958

Rabbit, fat infusion and exper. atherosclerosis, 247

Radioisotopes as indicators, 11, 113

Rat:

-, biochemistry of acute inflammation, 174

-, blood pressure, diminished lability, 47

-, changes in position of heart, 73 , consommation, eau et nourriture, 21

-, contraction thermique, tendons de la queue, 11

- $\quad$ - , affinité pour Ca45, Na24, K42, I131,

113

-, creatinuria and vitamin E, 139 
-, diurnal sexual periodicity (males), 64

-, electrocardiogram, 204

-, équilibre de Na et d'eau et labilité

tensionelle, 47 -, excretion urinaire, protéines, N-non-protéique, acide urique, créatinine, 33 -, life span, body weight and bone density on 10 different human diets, 185 -, metabolism and food consumption, 21 -, periarteriitis and genetics, 185 -, tail tendons and thermic contraction after pretreatment, 81 -, tendons de la queue et Ca45, 11 -, tooth decay and genetics, 185 -, urinary excretion of chloride, phosphate and urochrome, 123 -, urine et excrements, 21 -, ventricle weight changes, 204 Reaktionszeit, Unsicherheit der Lokali-sation optischer Reize und - (Homo), 284 Regeneration (Schwanz) bei Lebistes

retic. (Pisces), 266 RNA in der Entzündung (Ratte), 174 Saveur, sucrée et salée, seuil de perception, seuil de reconnaissance, 104 Sehnen, Rattenschwanz:

- $\quad$-, Affinität für Radioisotopen von $\mathrm{Ca}$,

Na, K und I bei thermischer Kon-

traktion, 113 , thermische Kontraktion nach Vor-

behandlung, 81

11 Sexual: , thermische Kontraktion und Ca45,

behaviour, diurnal periodicity, male rats, 64

difference, frequency of enlarged pituitary (rat), 185

Sexual:

, in tail regeneration Leb. ret.

(Pisces), 266

, threshold for recognition of salt -

iness (Homo), 104 Sinnesorgane, exponentielles Abfallen

der Leistung (Homo), 311 Sodium24, collagen fibres, affinity for -,

113 Steroide: -, bei experimenteller Atherosklerosis

(Kaninchen), 247

- $\quad$ und Gel-Faser-Verhältnis im Binde-

gewebe (Homo), 59

Tailregeneration, normal and retarded Leb. ret. (Pisces), 266

Tendon:

-, thermic contraction after pretreatment (rat, tail), 81

-, thermic contraction and Ca45 (rat, tail), 11

- $\quad$ thermic contraction and Ca45, Na24,

K42, and I131 (rat, tail), 113

Tissue élastique et collagène, aorte hu-

maine, 253 Urine: -, chloride, phosphate, and pigments

in-(rat), 123 -, creatin and créatinine excretion, and

vitamine $\mathrm{E}$ (rat), 139 -, production and water consumption

(rat), 21 -, protein, non-protein- $\mathrm{N}$, créatinine,

uric acid in - (rat), 33 -, steroid excretion and connective tissue (Homo), 59 Urochrome (rat),

123 Vision:

-, Perceptual difficulty (Homo), 1 -, Stimulus Exposure time (Homo), 1 -, Tachistoskopy

(Homo), 1 -, uncertainty and performance (Homo), 
284 Vitamins: -, B12, Plasmagehalt und Toleranztest

(Homo), 213 -, E und Alterskreatinurie (Ratte), 139 -, intake in urban and rural people (Switzerland), 321 Wasserhaushalt und Na-Stoffwechsel,

Einfluß auf Blutdruck (Ratte), 47 ZNS, degenerative Veränderungen und

Hörschwelle (Homo), 311 